\title{
Surface-Corrugated Microfiber Bragg Grating
}

\author{
Fei Xu, Jun-Long Kou, Yan-Qing Lu, Ming Ding and Gilberto Brambilla
}

Additional information is available at the end of the chapter

http://dx.doi.org/10.5772/52955

\section{Introduction}

Optical fiber Bragg gratings (FBG) are key devices for optical communication and sensors. FBGs are based on a periodic variation in the refractive index of the fiber core and allow for the reflection of a narrowband signal centered at a specific Bragg wavelength. Over the last two decades, FBGs have been manufactured mainly by modifying the core refractive index using interferometric or point-by-point techniques; most of interferometric techniques use a phase mask and an ultraviolet (UV) laser [1] (typically excimer or frequency doubled Ar+ ion) or femtosecond (fs) lasers (near IR [2] or UV [3]). Gratings based on surface corrugations have also been demonstrated in etched fibers using photolithographic techniques [4]. All gratings fabricated in thick fibers have weak refractive index modulations $\left(\Delta \mathrm{n}_{\bmod } \sim 10^{-4}-10^{-3}\right)$ and the related grating lengths are of the order of several millimeters.

Structural miniaturization is one of the current trends for achieving higher-bandwidth, faster response and higher-sensitivity. Experimentally, FBG miniaturization has been achieved in two steps. Firstly, the fiber has been tapered into a subwavelength-scale microfiber (MF), considered to be the basic element for miniature fiberized devices and subsystems [5]. Then strong refractive index modulations $\left(\Delta n_{\bmod }>10^{-1}\right)$ are induced. Large $\Delta n_{\bmod }$ can be obtained by alternating layers of different materials, one of which can be air. Although this process in normal optical fibers imposes the removal of large amounts of material (the propagating mode is confined at a depth $>50 \mu \mathrm{m}$ from the fiber surface), in fiber tapers and tips it only requires the removal of $\sim 10,000$ less matter because the propagating mode is confined by the silica/air interface in areas with micron size. A few techniques have been proposed to fabricate surface-corrugated fiber gratings (SCMGs), including photorefractive inscription using etching [6], femtosecond lasers [7, 8], and focused ion beam (FIB) [9-16]. So far, FIB is the most flexible and powerful tool for patterning, cross-sectioning or functionalizing a subwavelength circular microfiber due to its small and controllable spot size and high beam current density. In the past two years, a number of ultra-compact SCMGs have been successfully fabricated by FIB milling, with 
lengths as small as ten micrometers. In addition, a variety of other techniques have been proposed to generate gratings exploiting the fraction of power propagating in the evanescent field: these include wrapping a microfiber on a microstructured rod or put a microfiber on a surface-corrugated planar grating. This chapter reviews recent advances in ultra-small SCMGs, their characteristics and applications.

\section{Theory}

\subsection{SCMG spectral properties}

In SCMGs, strong $\Delta \mathrm{n}_{\bmod }$ is achieved alternating layers of air and glass. Figure 1 shows a typical SCMG geometry: the surface-corrugated structure is fabricated by inducing periodic microscale open-notches on the side or holes across the optical microfiber.

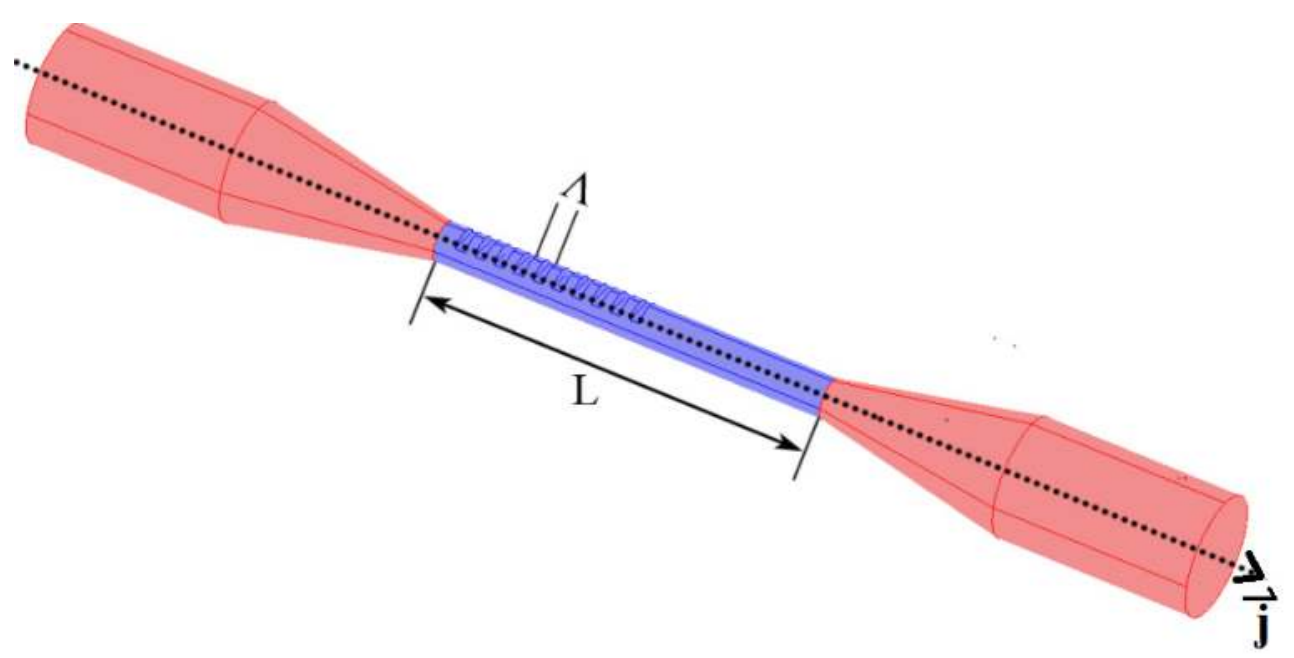

Figure 1. Schematic illustration of SCMGs. L and $\Lambda$ are the grating length and period, respectively. $\vec{j}$ is the unit vector along the fibre longitudinal axis.

In the grating, the forward (f) and backward (b) propagating modes are related by

$$
\overrightarrow{\beta_{b}}=\overrightarrow{\beta_{f}}+m \frac{2 \pi}{\Lambda} \vec{j}
$$

where $\beta_{i}=(2 \pi / \lambda) n_{\text {eff }, i}(i=f$ or $b)$ is the mode propagation constant, $m$ is the diffraction order, $\Lambda$ is the period of the grating and $\vec{j}$ is the unit vector along the fibre longitudinal axis. In a more physical perspective, Eq. (1) means that the momentum mismatch between the forward and backward propagating modes should be compensated by the reciprocal vector provided by the periodical index modulation. For the first-order diffraction which is commonly seen in SCMGs: 


$$
\left(n_{e f f, f}+n_{e f f, b}\right) \Lambda=\lambda_{B}
$$

Furthermore, if the two modes are identical, the commonly used Bragg resonance condition can be obtained, namely, $\lambda_{\mathrm{B}}=2 \mathrm{n}_{\text {eff }} \Lambda$.

For a SCMG, the effective index difference between the MF milled and un-milled cross sections can be as large as $\sim 10^{-3}[9]$, or even $\sim 10^{-1}[12]$, orders of magnitude larger than that in conventional FBGs. One way to calculate an averaged effective index of the grating region is to choose an unperturbed waveguide boundary using the method developed by W. Streifer [17]: the boundary between different materials is shifted to compensate for different geometry, shown as the dashed line in Fig. 2. $\mathrm{d}$ is the depth of the corrugation and heff is the boundary shift from the top of the corrugation to the new boundary of the corresponding unperturbed waveguide. The boundary shift heff illustrated in Fig. 2(b) and (c) is determined so that the volume bounded by the upper part of the corrugation $\left(\mathrm{S}_{\mathrm{A}} \tau\right)$ is equal to the volume bounded below $\left[\mathrm{S}_{\mathrm{B}}(1-\tau)\right]$, i.e.

$$
S_{A} \tau=S_{B}(1-\tau)
$$

where $\tau$ is the duty cycle. The averaged effective index could thus be obtained by mode analysis after the unperturbed waveguide boundary is established.

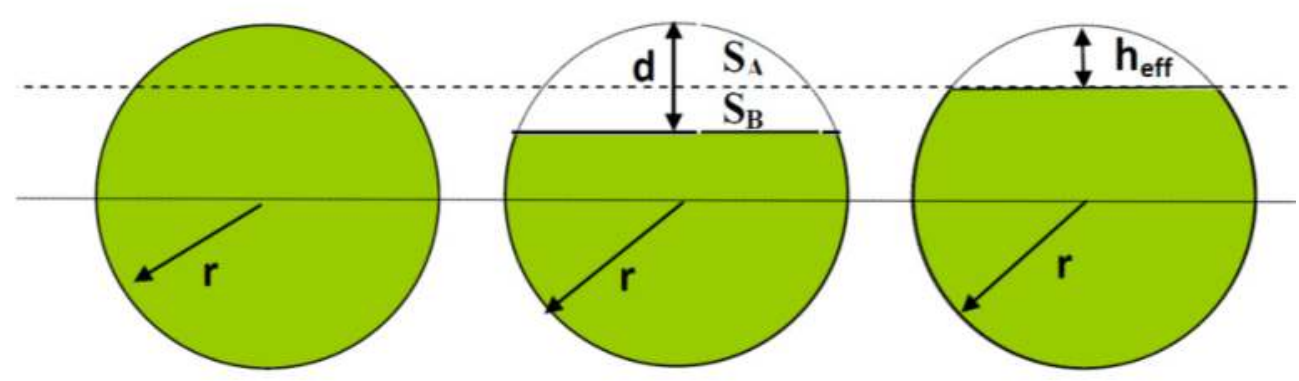

(a)

(b)

(c)

Figure 2. Equivalent unperturbed geometry. The cross-sections of (a) an un-milled MF, (b) a milled MF and (c) an equivalent unperturbed geometry, respectively. $d$ is the groove height and heff is effective height, obtained solving eq. 3 ..

The SCMG reflection spectrum can be estimated using [18]. However, due to the large index difference in the corrugation region, strong scattering may occur. A more effective way to verify the experimental spectrum obtained from the SCMG is a 3D finite element simulation, as shown in Fig. 3. This method takes the details of the structural deformation into consideration and thus better reflects the real situation experienced by the light field. 

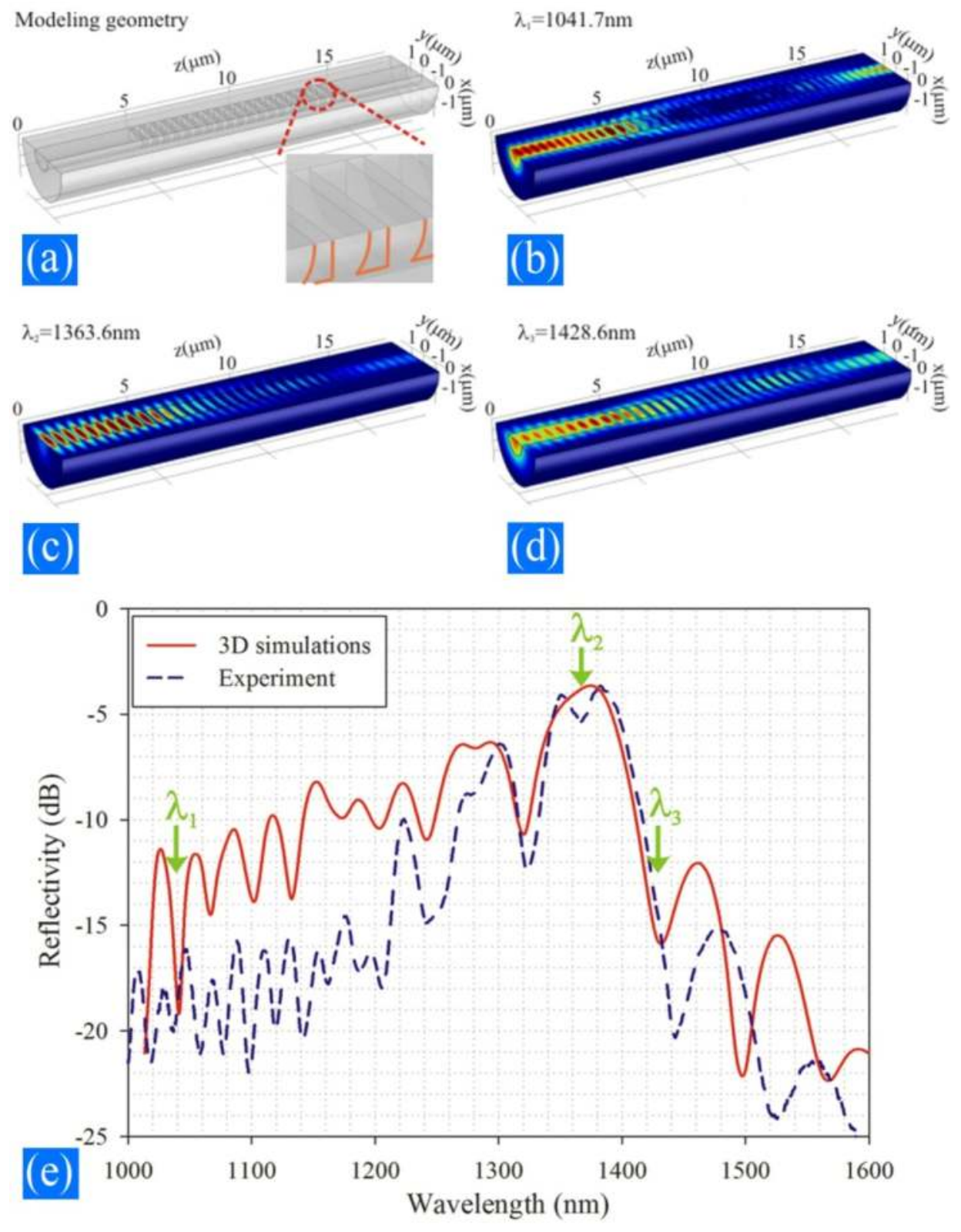

Figure 3. (a) Schematic of a 3D finite element simulation of SCMG; the insert shows the magnified figure of the biconcave air notch. (b), (c), and (d) Electric fields at wavelength $\lambda_{1}=1041.7 \mathrm{~nm}, \lambda_{2}=$ 1363.6nm, and $\lambda_{3}=1428.6 \mathrm{~nm}$, respectively. (e) SCMG reflection spectra. The red solid line is the 3D simulation line while the blue dashed line is the experiment result. $\lambda_{1}, \lambda_{2}$, and $\lambda_{3}$ represent the wavelengths whose electric fields are shown in (b), (c), and (d) [19]. 


\subsection{SCMG sensing mechanisms}

In the past years, FBGs have been widely applied to the measurement of chemical, biomedical, physical, electrical parameters, especially for structural health monitoring in civil infrastructures, where the information of measurands usually rely on the monitoring of the shift in wavelength of the reflected Bragg signal with the changes in the measurand (e.g., refractive index, temperature, and strain/force). FBG based sensors have a number of advantages with respect to conventional sensors, such as compactness, immunity to electromagnetic interference, multiplexing, rapid response to real time monitoring, and high sensitivity to external perturbations. Compared with conventional FBGs, SCMGs have similar applications and advantages with some extra benefits such as ultra-compact size and large evanescent field.

As the effective index and period of grating is a function of $r, n_{a}, T$, and $\varepsilon$, the Bragg condition (eq. 2) can be rewritten as [19]

$$
\lambda_{B}=2 n_{e f f}\left(r, n_{f}, n_{a}, T, \varepsilon\right) \Lambda(T, \varepsilon) .
$$

where the refractive index of fused silica and of the ambient medium surrounding the SCMGs are denoted by $\mathrm{n}_{\mathrm{f}}$ and $\mathrm{n}_{\mathrm{a}}, \mathrm{T}$ is the temperature and $\varepsilon$ is the strain applied to the SCMGs.

\subsubsection{Refractive index}

The notable distinction between SCMGs and conventional FBGs lies in the large SCMG evanescent field which enables its capabilities for external medium sensing. When a SCMG is operated as a refractive index (RI) sensor, the wavelength shift depends on the change of na. The sensor sensitivity $\left(\mathrm{S}_{\mathrm{a}}\right)$ with respect to the ambient medium $\mathrm{RI}$ is defined as [19]

$$
S_{a}=\frac{d \lambda_{B}}{d n_{a}}=\frac{\partial \lambda_{B}}{\partial n_{e f f}\left(n_{a}, r_{M F}\right)} \frac{\partial n_{e f f}\left(r_{M F}, n_{a}\right)}{\partial n_{a}}=2 \Lambda \frac{\partial n_{e f f}}{\partial n_{a}} .
$$

Figure 4 shows $S_{a}$ as a function of rMF. $n_{a}$ is chosen to be 1.33 and 1.42 , because most of the RI sensors work around these values. Sa increases for decreasing MF diameters, as larger fractions of power propagate in the evanescent field, thus in the surrounding environment. For the same reason, higher external RIs are associated with a larger $S_{a}$. Theoretically, the largest $S_{a}$ which can be obtained by SCMG is $\sim 2 \Lambda$, typically around $1100 \mathrm{~nm} / \mathrm{RIU}$ according to Eq. (5). This value is comparable to optical microfiber coil resonator sensor $[20,21]$ and higher than sensors based on microcapillary resonator [22] or photonic crystal microresonator [23].

\subsubsection{Temperature}

Temperature affects the Bragg wavelength shift through the thermo-optical effect and thermal expansion in three ways: index variation, MF radius variation and grating period 
change, each of which is represented in Eq. (6). The temperature sensitivity ( $\left.\mathrm{S}_{\mathrm{T}}\right)$ can be defined as [19]:

$$
S_{T}=\frac{d \lambda_{B}}{d T}=2 \Lambda\left(\sigma_{T} \frac{\partial n_{e f f}}{\partial n_{f}}+r \alpha_{T} \frac{\partial n_{\text {eff }}}{\partial r}+n_{e f f} \alpha_{T}\right)
$$

Here, $\sigma_{\mathrm{T}}\left(1.2 \times 10^{-5} /{ }^{\circ} \mathrm{C}\right)$ is the thermo-optical coefficient and $\alpha_{\mathrm{T}}\left(5.5 \times 10^{-7} /{ }^{\circ} \mathrm{C}\right)$ is the thermal expansion coefficient of fused silica. As thermal expansion contributes less than $2 \mathrm{pm} /{ }^{\circ} \mathrm{C}$ to the total sensitivity, it is generally neglected. ST resulting from the thermo-optical effect is $10-20 \mathrm{pm} /{ }^{\circ} \mathrm{C}$ and dominates in temperature sensing, which is in agreement with previous results obtained using fiber tip Fabry-Perot interferometers [24].

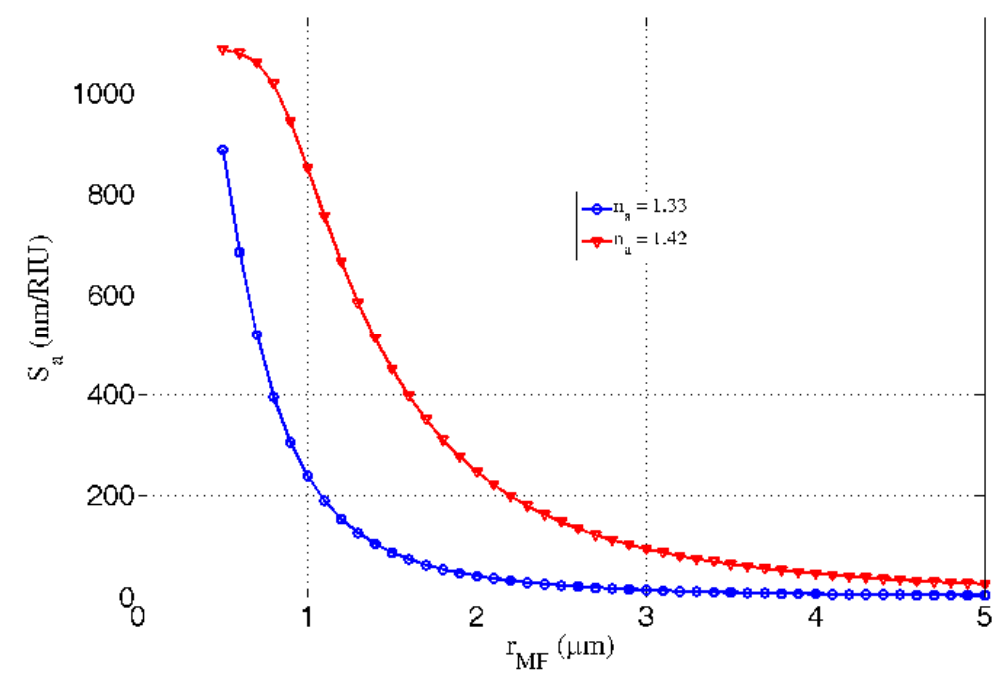

Figure 4. Dependence of sensor sensitivity $S_{a}$ on the MF radius rmF in the range $0.5-5 \mu m$ for two values of surrounding medium refractive indices na. Resonant wavelength is set at $1550 \mathrm{~nm}$ and na is chosen to be at 1.33 (blue circles) and 1.42 (red triangles). Only the fundamental mode is considered.

\subsubsection{Strain/Force}

From continuum mechanics, when longitudinal strain is applied to a SCMG, its Bragg wavelength shift can be estimated as follows [19]:

$$
\Delta \lambda_{B}=2 \Lambda n_{e f f}\left\{1-\left(\frac{n_{e f f}^{2}}{2}\right)\left[p_{12}-v\left(p_{11}+p_{12}\right)\right]\right\} \varepsilon=2 \Lambda n_{e f f}\left(1-p_{e f f}\right) \varepsilon
$$

where $\varepsilon$ is the applied strain, $v$ is Poisson's ratio and $\mathrm{p}_{\mathrm{ij}}$ coefficients are the Pockels' strainoptical tensor coefficients of the fiber material. If the MF cross section deformation due to the applied strain is neglected, strain sensitivity (Ss) is reduced to 


$$
S_{S}=\frac{\Delta \lambda_{B}}{\varepsilon}=\lambda_{B}\left(1-p_{e f f}\right)
$$

The effective photo-elastic coefficient peff for a SCMG strain sensor is $\sim 0.21$, giving $\mathrm{S} s \sim 1.2$ $\mathrm{pm} / \mu \varepsilon$, which is comparable to that of a conventional fiber grating with a Bragg wavelength of $1550 \mathrm{~nm}$. This is in agreement with experimental results [25, 26]. SCMG sensors can also be characterized by their force sensitivity $\left(\mathrm{S}_{\mathrm{F}}\right)$

$$
S_{F}=\frac{S_{S}}{\pi r_{M F}^{2} E},
$$

where E represents the fibre Young's modulus. Equation (9) shows that $S_{F}$ scales inversely with the square of the microfiber diameter.

For composite structures, like the SCMG wrapped on a PCF mentioned below, sensitivity depends on more parameters such as the strain-optical tensor of the photonic crystal fiber (PCF) support and the embedding coating.

\section{SCMG fabrication}

Several techniques have been reported in the literature for the fabrication of gratings in MFs and they can be classified as follows [19]:

1. Etch-eroded commercial FBG or UV irradiated FBG [25-33].

2. FIB-milled FBG on MFs [9-16].

3. Femtosecond-laser-irradiated FBG on MFs $[7,8]$.

4. FBGs exploiting evanescent fields [34-37].

Both cladding-etched commercial FBGs and UV irradiated FBGs in MFs are uniform microfiber gratings, meaning that the grating region experiences only RI modulation and not structural perturbations as indicated in Fig. 1. The most commonly used technique to get an uniform microfiber grating is to etch a single mode fiber (SMF) after the FBG has been written in the photosensitive Ge-doped core [25, 27-29]. Usually, a hydrofluoric acid aqueous solution $(\sim 20 \%-50 \%)$ at room temperature is employed for the etching process at an etching speed of $\sim 0.5-2 \mu \mathrm{m} / \mathrm{min}$. The diameter of the etched fiber can be measured and controlled in situ by monitoring the transmission loss.

Femtosecond-laser-irradiation is another way to induce periodical physical deformations on the surface of MFs [8]. During the femtosecond laser irradiation, the ultra-short laser pulses transfer energy to the electrons in the material irradiated through nonlinear ionization [38]. When a sufficiently high energy is achieved, pressure or shock waves cause melting or non-thermal ionic motion, resulting in permanent structural damages in the material. Aided with proper phase masks, uniform microfiber gratings can be fabricated on the surface of the MFs $[7,8]$.

For the fabrication of ultra-small SCMGs, the main technique is FIB milling or inducing external gratings. 


\subsection{FIB-milled SCMGs}

FIB milling, a powerful micromachining technique, has been the tool of choice to fabricate SCMGs [9-16]. This method employs accelerated ions to mill nanometer-scale features on MF surfaces to form corrugated structures. As the index modulation results from changes in the structure, this kind of gratings are called structural SCMGs.

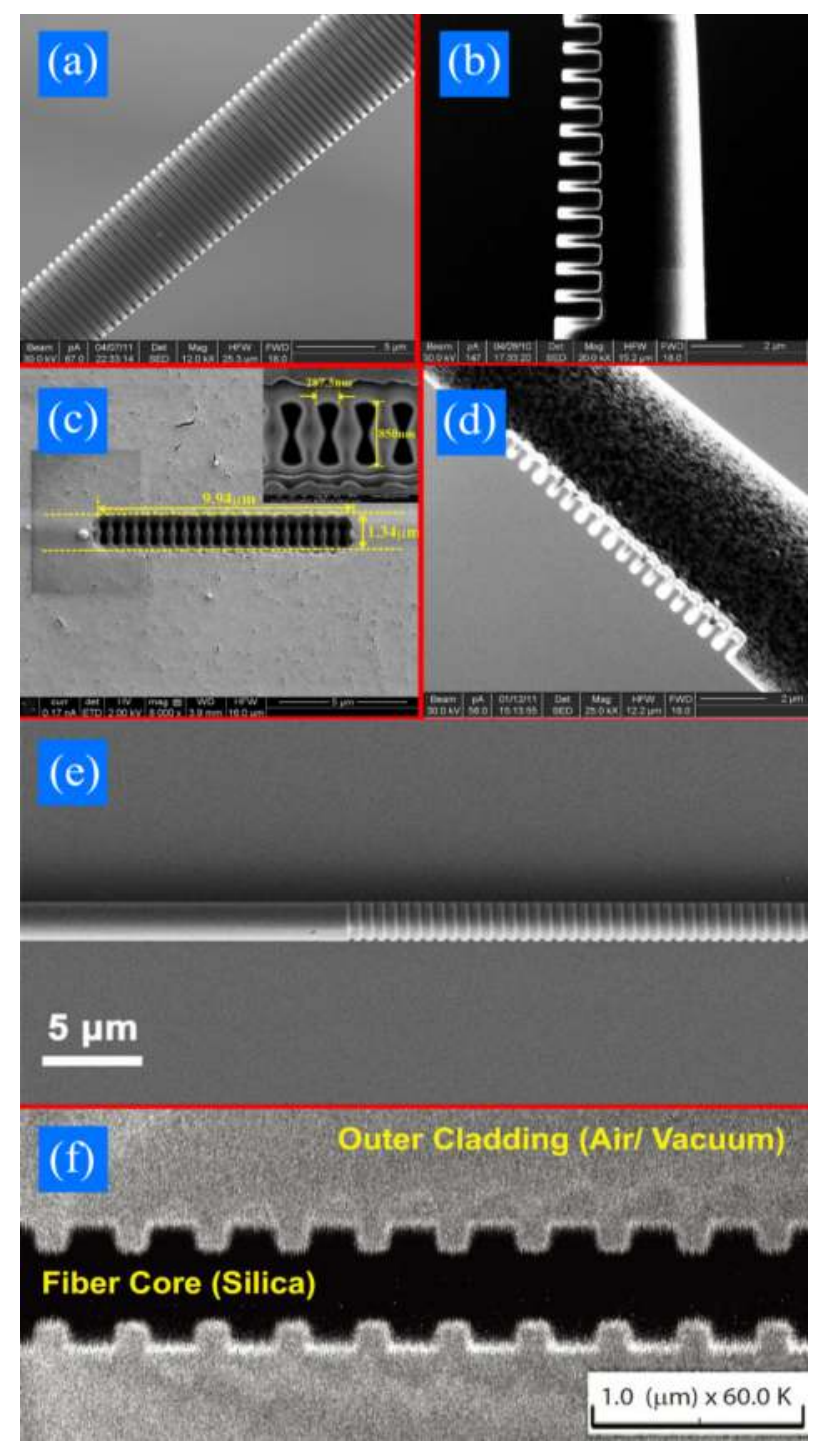

Figure 5. FIB/SEM pictures of gratings fabricated on MF tips (a)[9], (b)[10], (d)[13] and MF tapers (c)[12], (e)[14], (f)[15]. Reprinted with permission. Copyright 2011 Optical Society of America, Copyright 2012 AIP and Copyright 2011 IEEE. 
Prior to milling, the MF is coated with a thin film of metal, e.g. aluminum or gold [9-13], to prevent charge accumulation which cause ion deflections and large fabrication errors. Alternatively, MF can also be laid on a doped silicon wafer [14]: due to van der Waals' forces, the MF tightly attaches to the conductive substrate and it avoids charging by transferring charges to the wafer.

During the FIB micromachining process, the MF sample should be fixed firmly in the vacuum chamber to minimize sample displacements. A $30 \mathrm{kV}, 10-300 \mathrm{pA} \mathrm{Ga}{ }^{+}$ion beam is usually used to get a good milling accuracy. The total milling process takes minutes to hours according to the beam current used and milled area. After the machining process, when metal coating is not required, the MF is immersed in metal etchant to totally remove the metal film and then is cleaned with deionized water.

Figure 5 shows FIB/SEM pictures of SCMGs fabricated from different groups. Gratings in Fig. 5(a), (b), and (d) are fabricated on MF tips while the rest are on tapers. SCMGs are fabricated on MFs with diameter ranging from $560 \mathrm{~nm}$ [Fig. 5(f)] to $6.6 \mu \mathrm{m}$ [Fig. 5(a)] and the

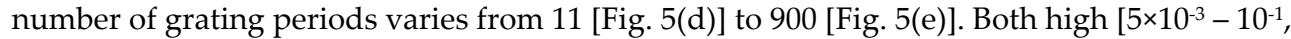
Fig. 5(c), (d), (f)] and low [10-4 $-5 \times 10^{-3}$, Fig. 5(a), (b), (e)] average RI modulations have been achieved by FIB-milled SCMGs. In all, FIB provides researchers a flexible way to get all kinds of structures with high accuracy at will and without additional masks. Yet, batch production cannot be envisaged for this method.

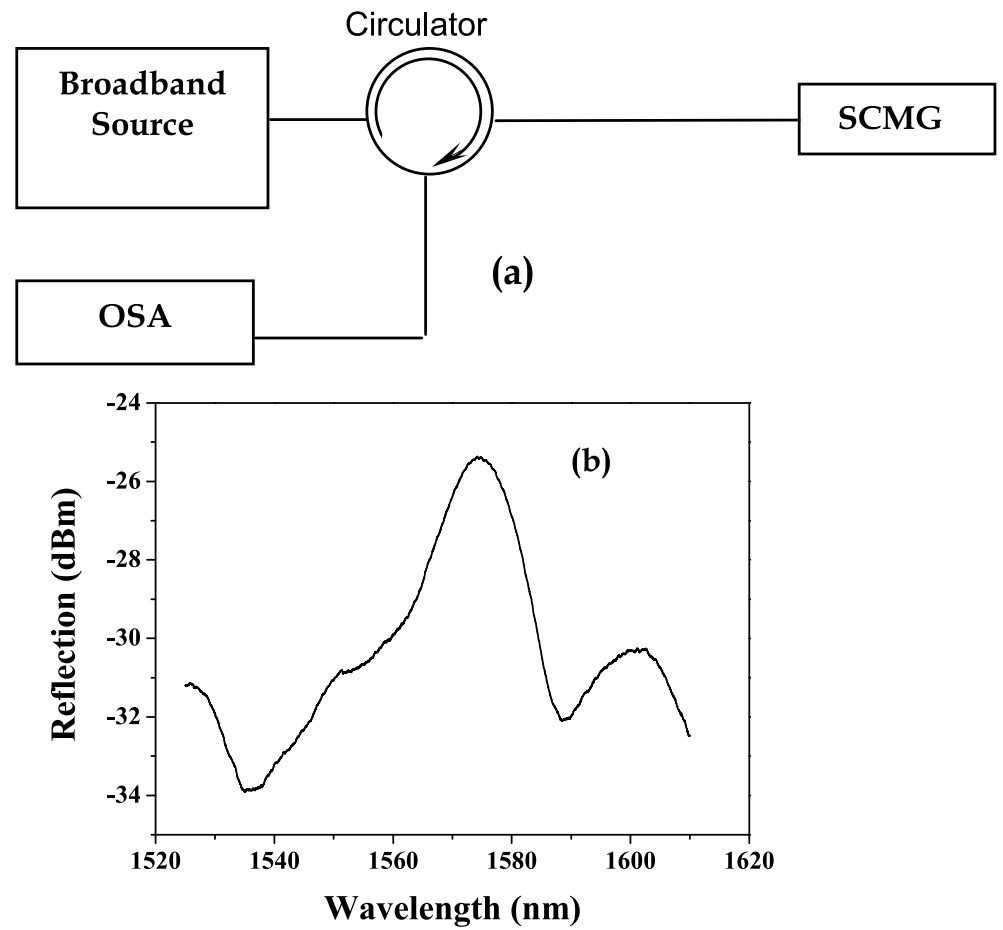

Figure 6. (a) Experimental set-up of characterizing SCMGs. (b) Reflection spectra of a SCMG in air. 
Experimentally, SCMGs are characterized with a set-up similar to that shown in Fig. 6(a): light from a broadband source is injected into the grating and the reflected light is then collected by an optical spectrum analyzer (OSA) (Ando AQ6317B, Japan) after passing through a circulator. The fiber tip reflection has been analyzed before grating inscription and displayed a negligible reflection over the whole spectrum, showing that the reflection at the tip end was insignificant. Fig. 6(b) shows the typical reflection spectrum of a SCMG. There is a clear peak at $\sim 1572 \mathrm{~nm}$ with an extinction of $10 \mathrm{~dB}$. Two side lobes are observed at longer $(1600 \mathrm{~nm})$ and shorter $(1535 \mathrm{~nm})$ wavelengths. The possible explanation of the wide bandwidth of the experiment reflection spectrum can be the effective indices variation, rough notch surfaces and departure from perfect grating periodicity.

\subsection{SCMG exploiting evanescent fields}

In addition to the previous techniques, other methods have demonstrated or proposed the fabrication SCMGs using the strong microfiber evanescent field. SCMGs can be manufactured by wrapping a MF on a microstructured rod and then coating them with a low-loss polymer. The rod can be obtained by etching a cane used in the manufacture of microstructured fibers or it can be a thick microstructured fiber. Here no expensive mask, laser or machining set-up is used. Combining current enabling technologies on microstructured optical fibers [39, 40] and MFs [41], it is possible to induce periodic or variable corrugations leading to the coupling between forward and backward propagating waves. The final SCMG structure is shown in Fig. 7(a) [34, 37]: it is a compact and strong micro-device with some air holes arranged in a circle. Fig. 7(b) presents a possible rod crosssection. The mode propagating in the microfiber experiences refractive index corrugations because its evanescent field overlaps with the support rod where there is alternation of glass and air holes. Here we only consider one ring of air holes; the inner large hole is also filled with air and the MF is assumed perpendicular to the rod. When a coordinate increasing along the microfiber is used, the surface corrugations experienced by the mode propagating along the curvilinear coordinate are similar to those experienced by a mode propagating straight in proximity of a conventional planar grating. Unfolding the MF, the MF grating can be taken as a coated MF on a planar substrate with air-hole corrugations: the equivalent structure is shown in Fig. 7(c) [34, 37].

The mode field in the unperturbed waveguide geometry is derived from the perturbed (straight) geometry using the method mentioned in section 2.1. If $\mathrm{g}_{1}$ is the boundary between the materials with refractive indices $n_{1}$ (air) and $n_{2}$ (the rod material, generally silica) and $g_{2}$ is the boundary between the materials with refractive indices $n_{4}$ (coating) and $\mathrm{n}_{2}$, a new $\mathrm{g}_{0}$ is chosen to match the volume of $\mathrm{n}_{1}$ material extending into region $\mathrm{A}$ (above $\mathrm{g}_{0}$ ) with the volume of $n_{2}$ material extending into region B (below go), as shown in Fig. 8(a). We introduce the effective distance deff between $g_{2}$ and $g_{0}$ as[34, 37]:

$$
d_{e f f}=g_{1}+g_{2}-g_{0}=d_{1}+d_{2}+2 r-\pi r^{2} / \Lambda
$$

$\mathrm{d}_{\text {eff }}$ increases for increasing $\mathrm{d}=\mathrm{d}_{1}+\mathrm{d}_{2}$ and increasing $\Lambda$. 


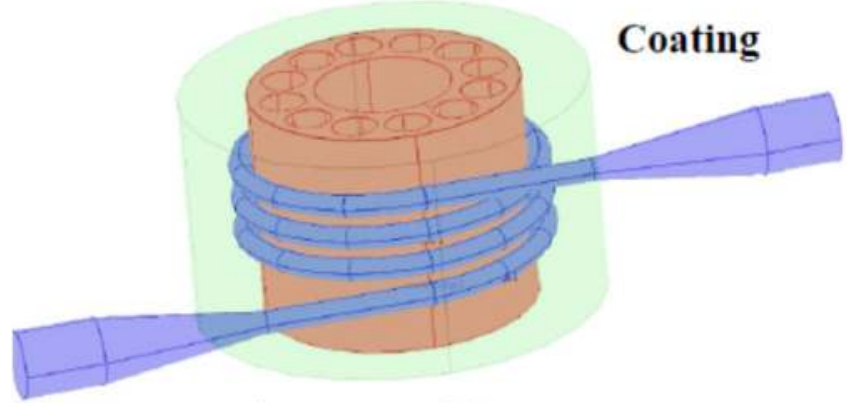

(a)

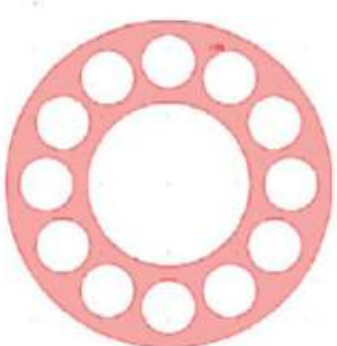

(b)

MF

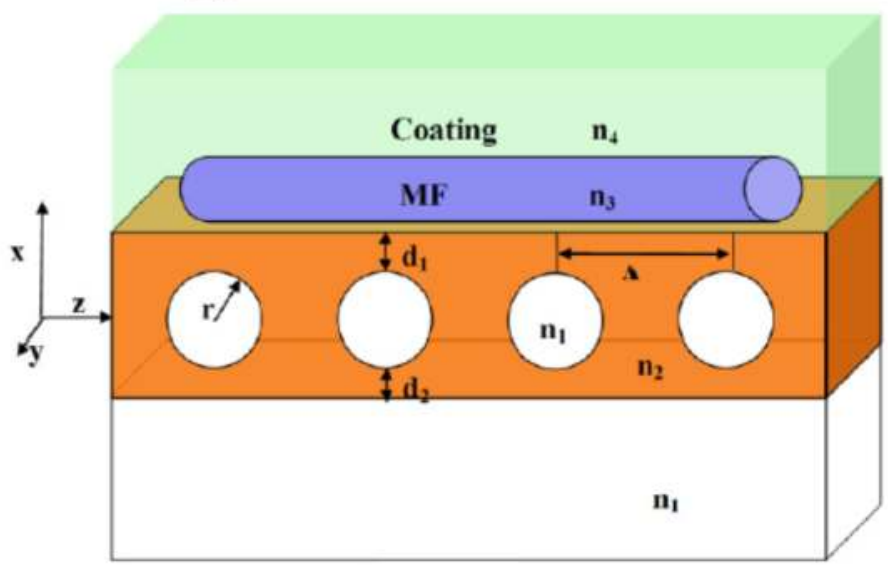

(c)

Figure 7. (a) Schematic of the embedded SCMG. (b) Cross-section of the support rod (c) the equivalent planar structure, $\mathrm{n}_{1}, \mathrm{n}_{2}, \mathrm{n}_{3}, \mathrm{n}_{4}$ are the refractive indices of the hole, the rod, the MF, and the coating, respectively[34, 37]. Copyright 2009 Optical Society of America and Copyright 2010 IEEE.

This method is also extremely flexible and can be used to manufacture chirped MF gratings: chirping can be achieved by tuning the hole radius and pitch, or by changing the distance between MF and holes. Since $\lambda=2 n_{\text {neff }}\left(d_{\text {eff }}\right) \Lambda / m$, there are three simple ways to realize chirp: by tuning $\Lambda, \mathrm{d}=\mathrm{d}_{1}+\mathrm{d}_{2}$, and $\mathrm{r}$. Chirp rates are defined as [34, 37]:

$$
\left\{\begin{array}{l}
\frac{\partial \lambda}{\partial \Lambda}=\frac{2}{m} \frac{\partial n_{\text {neff }}}{\partial d_{\text {eff }}} \frac{\pi r^{2}}{\Lambda}+\frac{2}{m} n_{\text {neff }} \\
\frac{\partial \lambda}{\partial r}=\frac{2}{m} \frac{\partial n_{\text {neff }}}{\partial d_{\text {eff }}}(2 \Lambda-2 \pi r) \\
\frac{\partial \lambda}{\partial d}=\frac{2}{m} \frac{\partial n_{\text {neff }}}{\partial d_{\text {eff }}} \Lambda
\end{array}\right.
$$


All the derivatives $\partial \lambda / \partial \Lambda, \partial \lambda / \partial d$ and $\partial \lambda / \partial r$ depend on $\partial n_{n e f f} / \partial d_{\text {eff, }}$ which in turn is strongly dependent on $\mathrm{d}_{\text {eff }}$ and $\mathrm{r}$. $\partial \mathrm{n}_{\text {neff }} / \partial \mathrm{d}_{\text {eff }}$ is of the order of $10^{-2} \mu \mathrm{m}^{-1}$ and can achieve the maximum at deff $\sim 1 \mu \mathrm{m}$. For $\mathrm{m}=2$, from Eq. (11) $\partial \lambda / \partial \Lambda \gg \partial \lambda / \partial d \sim \partial \lambda / \partial$; since $\partial \lambda / \partial \Lambda>1$, chirped grating can be easily realized by tuning the grating period: current PCF fabrication techniques make the low-cost period-tuning possible. Moreover, $\partial \lambda / \partial \mathrm{d} \sim \partial \lambda / \partial \mathrm{r} \sim 10^{-5}$ : which is enough to produce small precise chirps in a simpler and cheaper way than inducing a temperature, strain or refractive-index gradients $[34,37]$.
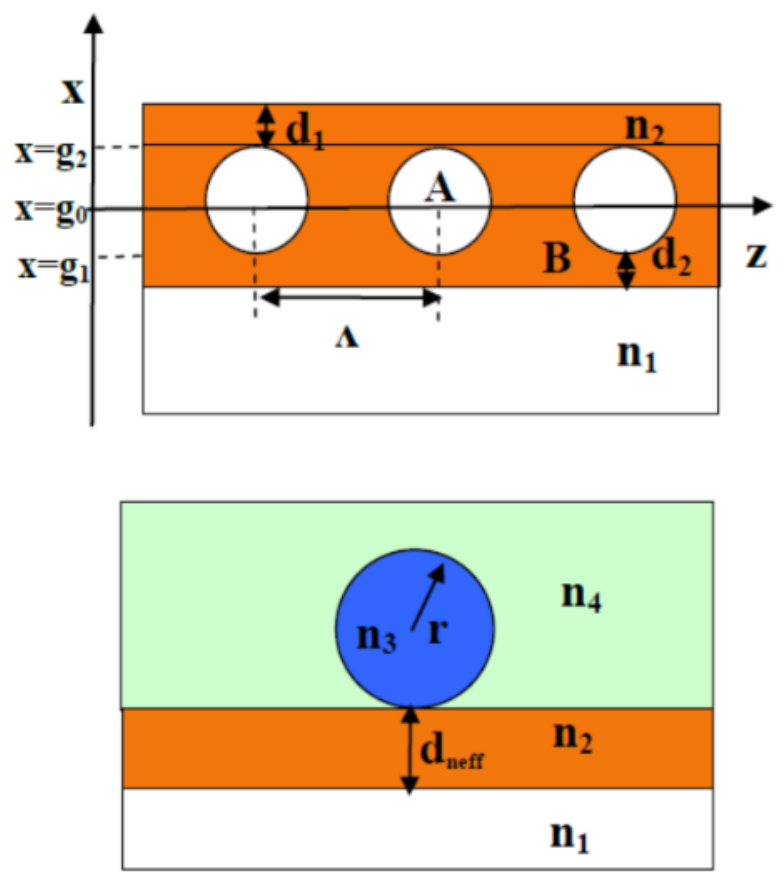

Figure 8. Top: Illustration of the outer layer structure of the support rod in Streifer's theory. $\mathrm{n}_{1}$ and $\mathrm{n}_{2}$ represent the refractive indices of the hole and rod, $\Lambda$ the distance between two adjacent holes, $g_{1}$ and $g_{2}$ the boundaries between different layers and go represents the new equivalent boundary between $\mathrm{n}_{2}$ and $\mathrm{n}_{1}$. Bottom: cross-section of the MF in the equivalent outer straight layer structure; $\mathrm{n}_{3}$ and $\mathrm{n}_{4}$ are the indices of the MF and coating; $r$ is the radius of the MF and deff is the effective wall thickness[34, 37]. Copyright 2010 Optical Society of America and Copyright 2009 IEEE.

These methods to induce chirp mainly depend on the geometry of the support rod; in fact hole pattern has a periodicity related to the MF turn around the support rod, the circumference of which limits the chirp length. An alternative method to achieve chirped gratings relies on tilting the MF with respect to the support rod longitudinal axis: if the MF cross the rod at an angle $\phi$ the Bragg condition is expressed by [42] $\lambda=2 n_{\text {eff }} \Lambda / \cos \varphi / m$; by changing $\phi$ gradually, the Bragg wavelength changes gradually, it is possible to control the grating Bragg wavelength and get a chirped grating. When $\phi$ is very small, the chirp rate is given by [34, 37] 


$$
\frac{\partial \lambda}{\partial \varphi}=-2 \frac{n_{e f f} \Lambda \sin \varphi}{m \cos ^{2} \varphi} \approx 2 \frac{n_{e f f} \Lambda \varphi}{m}=\lambda \varphi
$$

The chirp range varies considerably: from $0.3 \mathrm{~nm} /{ }^{\circ}$ at $\phi=0.1^{\circ}$ to $300 \mathrm{~nm} /{ }^{\circ}$ at $\phi=10^{\circ}$. These two chirping methods allow for a great deal of flexibility in the chirp design[34, 37].

The use of microstructured support rods to make gratings provides an extreme flexibility because it is easier to be dealt with than the microfiber itself. By controlling the air holes geometry, it is possible to get several-layer corrugations or phase shifted gratings. If the rod is coated with an active layer or the holes are filled with an active medium, it generates a laser. If holes are used as microfluidic channels, the support rod can work as a sensor. Moreover, since only a very short piece of rod is needed for each device, the average device cost is very low. The device can be coated with stable polymer such as Teflon and UV375 and keep it very strong [34,37].

A similar method relies on laying the MF on a substrate with pre-treated microstructures [see Fig. 9]. The fraction of power propagating in the evanescent field interacts with the periodically distributed patterns in the rod or the substrate, and light transmission can then be modulated. Both methods avoid post-processing the thin MFs and have great flexibility. However, the MFs have to be coated with low index polymer [43] which means that they are not suitable for high temperature sensing.

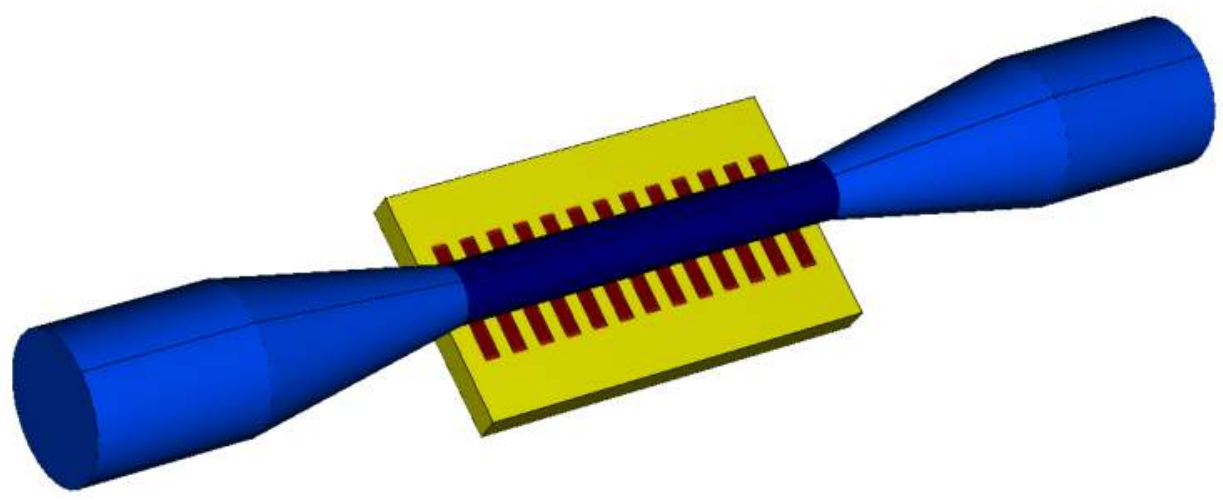

Figure 9. Proposed SCMG by laying the MF on a substrate with pre-treated microstructures[44].

Finally, Ding et al.[35] combined metal lift-off technology with lithography to produce metallic surface gratings, which provided a high and constant sensitivity to the ambient medium RI, while Phan Huy et al. [36] demonstrated an improvement in the sensitivity of RI by making use of the suspended core of a microstructured fiber.

\section{Sensing applications}

SCMGs can have a number of possible sensing applications as conventional FBGs. Up to date, SCMGs have been used to measure refractive index, temperature and strain/force. 
With the extreme small size and flexible geometry, SCMGs offer great prospects for developing novel sensors with a very small perturbation on the object being measured.

\subsection{Refractive index sensing}

Much of the SCMG applications relate to RI sensing because of the very large MF evanescent field. For a typical SCMG sensor immersed in ambient liquid with RI in the range $1.32-1.46$, $\mathrm{S}_{\mathrm{a}}$ varies from $10 \mathrm{~nm} / \mathrm{RIU}$ (refractive index unit) to $10^{3} \mathrm{~nm} / \mathrm{RIU}$, according to the MF radius and the ambient liquid sensed, whatever for a FIB-milled or rod-wrapped SCMG. Usually, a smaller radius and a larger ambient medium RI result in a higher sensitivity regardless of the fabrication method. For example, Liang et al. got a sensitivity of $16 \mathrm{~nm} / \mathrm{RIU}$ at a RI around 1.35 with a MF $6 \mu \mathrm{m}$ in diameter [27] while $660 \mathrm{~nm} / \mathrm{RIU}$ was reached by Liu et al. at a RI of 1.39 by using a $1.8 \mu \mathrm{m}$-diameter MF [14]. Both of them agree well with what is predicted from Eq. (5).

In addition to all these nonmetallic SCMGs, metallic gratings have also been proposed for RI sensing. The existence of metal causes light to be coupled to modes of different properties $[10,35]$. Figure 10(a) shows the reflection spectra of the metal-dielectric-hybrid SCMG [Fig. 5(d)] immersed in air, acetone, and isopropanol, respectively. The extinction ratio is about $10 \mathrm{~dB}$. The small degree of chirp has been ascribed to the non-uniformity of the taper. The taper diameter difference at the grating extremities is less than $1 \mu \mathrm{m}$ as illustrated in Fig. 5(d). According to simulations, different effective refractive indices resulting from the variation of the diameter induce resonant wavelength shifts of $\sim 4 \mathrm{~nm}$. Some small ripples in Fig. 10(a) can probably be due to a degree of chirping [10].

The grating spectrum presents several valleys and peaks with different characteristics in a $100 \mathrm{~nm}$ wide spectral range [10]. The peaks shift when the outer environment changes from acetone to isopropanol. However, these valleys and peaks show larger shifts at longer wavelengths, while those at shorter wavelengths shift much less and almost stop at specific wavelengths, meaning that the reflected light can be coupled to different modes. In the micrometer-diameter metal-dielectric-hybrid fiber tip, several modes are probably excited with close propagation constant because of the metal cladding [45]. Some modes are well confined in the tip and have negligible field overlap with the liquid while other modes are not. The different valleys and peaks correspond to the coupling between these different forward and backward propagating modes, with different response properties to any outer environment change [10].

The metal-dielectric-hybrid grating showed RI sensitive (a in Fig. 10(a)) and insensitive (d in Fig. 10(a)) behavior for different resonant modes [10]. Sa of the sensitive channel (125 $\mathrm{nm} / \mathrm{RIU}$ ) is one order of magnitude larger than that of a nonmetallic SCMG with the same radius whereas $S_{a}$ of the insensitive channel $(8 \mathrm{~nm} / \mathrm{RIU})$ is one order of magnitude smaller. This can be attributed to the fact that the introduction of metal film causes the MF to support both surface guided modes (which have a larger modal overlap with the ambient medium, a and $b$ in Fig. 10) and bound modes (where most of the energy is located in the dielectric core, $c$ and $d$ in Fig. 10). The smallest sensitivity can be further decreased to nearly zero by 

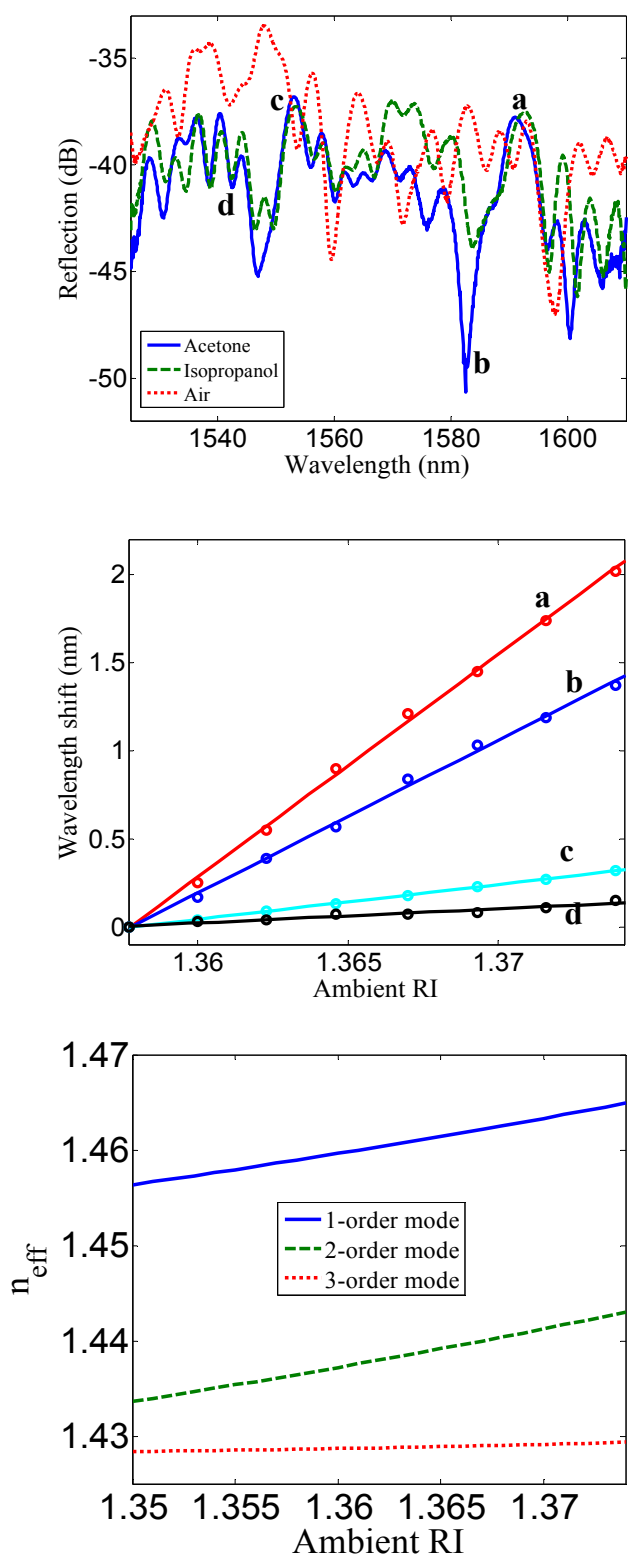

Figure 10. Top: measured reflection spectra of the metal-dielectric-hybrid grating when immersed in air, acetone, and isopropanol. a, b, c, d denote different peaks and valleys labelled. Centre: Dependence of wavelength shift on ambient RI for different modes in a metal-dielectric-hybrid SCMG. Bottom: Calculated effective index of one cladding mode and one core mode as a function of the outer liquid refractive index na. The radius of the fiber tip is assumed to be $3 \mu \mathrm{m}$ with a golden coating $20 \mathrm{~nm}$ thick [10]. Copyright 2011 IEEE. 
optimizing the tip grating profile and metal coating. Because of many different properties on the outer liquid refractive index, the metal-dielectric-hybrid grating can be applied as a multi-parameter sensor and the index-insensitive channel can be used to simultaneously measure temperature, pressure, and so on [10].

\subsection{Temperature sensing}

Although thermal post-processing and hydrogen loading have been shown to induce grating capable of standing temperatures as high as $1300{ }^{\circ} \mathrm{C}$ in conventional fibers [46], in MF thermometers, up to now, only SCMGs without polymer have been reported operating above $200{ }^{\circ} \mathrm{C}[9,13]$. The sensitivity of these components is around $20 \mathrm{pm} /{ }^{\circ} \mathrm{C}$, similar to the value predictable using Eq. (6). Figure 11 is the experimental characterization of the FIB milled SCMG demonstrated using the sample shown in Fig. 5(a). As the temperature increases, the Bragg wavelength red shifts. The extremely short SCMG length $(\sim 36.6 \mu \mathrm{m})$ and wide operating range $\left(\sim 20-450^{\circ} \mathrm{C}\right)$ presents it as a promising candidate for detecting temperature change in ultra-small space.

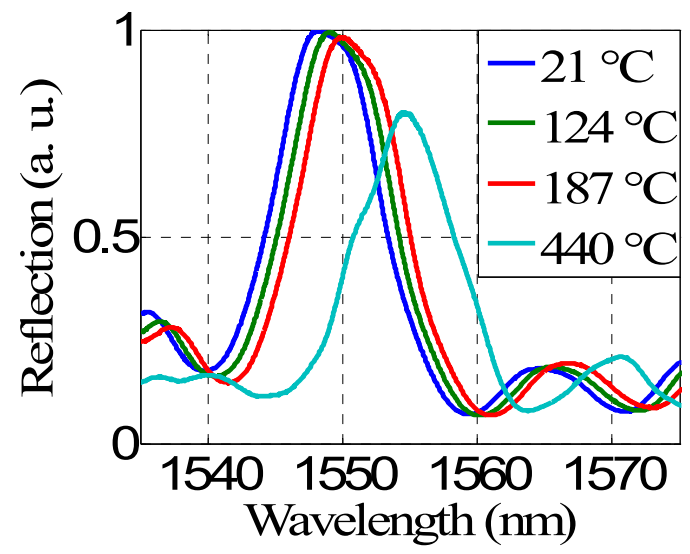

Figure 11. Reflection spectra of the FIB-milled SCMG in air at four different temperatures.

\subsection{Strain/Force sensing}

Although Ss remains almost the same for different MF diameters[26], SF varies with the MF radius according to Eq. (9). A SCMG with diameter of $3.5 \mu \mathrm{m}$ reaches a force sensitivity of $1900 \mathrm{~nm} / \mathrm{N}$, which is more than three orders of magnitude compared to that of a conventional fibers [26]. If the Bragg wavelength can be detected to an accuracy of $0.05 \mathrm{~nm}$, forces in the order of $10^{-5} \mathrm{~N}$ can be measured. For the sample reported in Fig. 5(d), where the silica constitute only a small fraction of the SCMG cross section, a further three orders of magnitude improvement in sensitivity is predicted, with $S_{F}$ reaching values in excess of $10^{6}$ $\mathrm{nm} / \mathrm{N}$, corresponding to forces of the order of $\mathrm{nN}$. The SCMG strain/force sensors could offer attractive properties monitoring strain/force changes in power plant pipelines, airplane wings, and other civil engineering structures [19]. 


\section{Conclusions}

This review presented the fabrication, operating principles and applications of surface corrugated microfiber Bragg gratings (SCMGs). SCMGs can potentially outperform conventional FBGs because of their large evanescent field and compactness. Taking advantage of their extreme small size and unique geometry, SCMGs exhibit beneficial sensor properties such as fast response, high resolution and small-objection detections. SCMGs could find promising sensing applications in detecting parameter variations in ultra-small space [19].

\section{Acronyms}

$\begin{array}{ll}\text { FBG } & \text { fiber Bragg gratings } \\ \text { FIB } & \text { focused ion beam } \\ f_{S} & \text { femtosecond } \\ \text { MF } & \text { microfiber } \\ \text { OSA } & \text { optical spectrum analyzer } \\ \text { SCMG } & \text { surface-corrugated fiber grating } \\ \text { SMF } & \text { single mode fiber } \\ \text { UV } & \text { ultraviolet }\end{array}$

\section{Author details}

Fei $\mathrm{Xu}$, Jun-Long Kou and Yan-Qing Lu

College of Engineering and Applied Sciences and National Laboratory of Solid State Microstructures, Nanjing University, Nanjing, P. R. China

Ming Ding and Gilberto Brambilla

Optoelectronics Research Centre, University of Southampton, Southampton, SO17 1BJ,

United Kingdom

\section{Acknowledgement}

F. Xu and Y.-q Lu acknowledge the support from National 973 program under contract No. 2011CBA00200 and 2012CB921803, NSFC program No. 11074117 and 60977039, and the Priority Academic Program Development of Jiangsu Higher Education Institutions (PAPD). G. Brambilla gratefully acknowledges the Royal Society (London, U.K.) for his University Research Fellowship.

\section{References}

[1] K. O. Hill, B. Malo, F. Bilodeau, D. C. Johnson, and J. Albert, "Bragg gratings fabricated in monomode photosensitive optical fiber by UV exposure through a phase mask " Appl. Phys. Lett. 62, 1035-1037 (1993). 
[2] S. J. Mihailov, C. W. Smelser, P. Lu, R. B. Walker, D. Grobnic, H. M. Ding, G. Henderson, and J. Unruh, "Fiber Bragg gratings made with a phase mask and 800-nm femtosecond radiation," Opt. Lett. 28, 995-997 (2003).

[3] S. A. Slattery, D. N. Nikogosyan, and G. Brambilla, "Fiber Bragg grating inscription by high-intensity femtosecond UV laser light: comparison with other existing methods of fabrication," J. Opt. Soc. Am. B-Opt. Phys. 22, 354-361 (2005).

[4] C. Y. Lin and L. A. Wang, "A wavelength- and loss-tunable band-rejection filter based on corrugated long-period fiber grating," IEEE Photon. Technol. Lett. 13, 332-334 (2001).

[5] G. Brambilla, F. Xu, P. Horak, Y. Jung, F. Koizumi, N. P. Sessions, E. Koukharenko, X. Feng, G. S. Murugan, J. S. Wilkinson, and D. J. Richardson, "Optical fiber nanowires and microwires: fabrication and applications," Adv. Opt. Photon. 1, 107-161 (2009).

[6] T. L. Lowder, K. H. Smith, B. L. Ipson, A. R. Hawkins, R. H. Selfridge, and S. M. Schultz, "High-Temperature Sensing Using Surface Relief Fiber Bragg Gratings," IEEE Photonic. Technol. Lett. 17, 1926-1928 (2005).

[7] D. Grobnic, S. J. Mihailov, D. Huimin, and C. W. Smelser, "Bragg grating evanescent field sensor made in biconical tapered fiber with femtosecond IR radiation," IEEE Photon. Technol. Lett. 18, 160-162 (2006).

[8] X. Fang, C. R. Liao, and D. N. Wang, "Femtosecond laser fabricated fiber Bragg grating in microfiber for refractive index sensing," Opt. Lett. 35, 1007-1009 (2010).

[9] J.-1. Kou, S.-j. Qiu, F. Xu, and Y.-q. Lu, "Demonstration of a compact temperature sensor based on first-order Bragg grating in a tapered fiber probe," Opt. Express 19, 1845218457 (2011).

[10] J.-1. Kou, S.-j. Qiu, F. Xu, Y.-q. Lu, Y. Yuan, and G. Zhao, "Miniaturized metal-dielectrichybrid fiber tip grating for refractive index sensing," IEEE Photon. Technol. Lett. 23, 1712-1714 (2011).

[11] M. Ding, P. Wang, T. Lee, and G. Brambilla, "A microfiber cavity with minimal-volume confinement," Appl. Phys. Lett. 99, 051105 (2011).

[12] M. Ding, M. N. Zervas, and G. Brambilla, "A compact broadband microfiber Bragg grating," Opt. Express 19, 15621-15626 (2011).

[13] J. Feng, M. Ding, J.-1. Kou, F. Xu, and Y.-q. Lu, "An optical fiber tip micrograting thermometer," IEEE Photon. J. 3, 810-814 (2011).

[14] Y. Liu, C. Meng, A. P. Zhang, Y. Xiao, H. Yu, and L. Tong, "Compact microfiber Bragg gratings with high-index contrast," Opt. Lett. 36, 3115-3117 (2011).

[15] K. P. Nayak, F. Le Kien, Y. Kawai, K. Hakuta, K. Nakajima, H. T. Miyazaki, and Y. Sugimoto, "Cavity formation on an optical nanofiber using focused ion beam milling technique," Opt. Express 19, 14040-14050 (2011).

[16] F. L. Kien, K. P. Nayak, and K. Hakuta, "Nanofibers with Bragg gratings from equidistant holes," J. Mod. Opt. 59, 274-286 (2012).

[17] K. Handa, S. Peng, and T. Tamir, "Improved perturbation analysis of dielectric gratings," Applied Physics A: Materials Science \& Processing 5, 325-328 (1975).

[18] T. Erdogan, "Fiber grating spectra," IEEE J. Lightwave Technol. 15, 1277-1294 (1997).

[19] J.-L. Kou, M. Ding, J. Feng, Y.-Q. Lu, F. Xu, and G. Brambilla, "Microfiber-Based Bragg Gratings for Sensing Applications: A Review," Sensors 12, 8861-8876 (2012). 
[20] F. Xu, P. Horak, and G. Brambilla, "Optimized Design of Microcoil Resonators," Journal of Lightwave Technology 25, 1561-1567 (2007).

[21] F. Xu, P. Horak, and G. Brambilla, "Optical microfiber coil resonator refractometric sensor: erratum," Opt. Express 15, 9385-9385 (2007).

[22] I. M. White, H. Zhu, J. D. Suter, N. M. Hanumegowda, H. Oveys, M. Zourob, and X. Fan, "Refractometric sensors for lab-on-a-chip based on optical. ring resonators," Ieee Sensors Journal 7, 28-35 (2007).

[23] M. Adams, G. A. DeRose, M. Loncar, and A. Scherer, "Lithographically fabricated optical cavities for refractive index sensing," Journal of Vacuum Science \& Technology B 23, 3168-3173 (2005).

[24] J.-1. Kou, J. Feng, L. Ye, F. Xu, and Y.-q. Lu, "Miniaturized fiber taper reflective interferometer for high temperature measurement," Opt. Express 18, 14245-14250 (2011).

[25] L. Sang-Mae, S. S. Saini, and J. Myung-Yung, "Simultaneous measurement of refractive Index, temperature, and strain using etched-core fiber Bragg grating sensors," IEEE Photon. Technol. Lett. 22, 1431-1433 (2010).

[26] T. Wieduwilt, S. Bruckner, and H. Bartelt, "High force measurement sensitivity with fiber Bragg gratings fabricated in uniform-waist fiber tapers," Meas. Sci. Technol. 22, 075201 (2011).

[27] W. Liang, Y. Huang, Y. Xu, R. K. Lee, and A. Yariv, "Highly sensitive fiber Bragg grating refractive index sensors," Appl. Phys. Lett. 86, 151122 (2005).

[28] A. Iadicicco, S. Campopiano, A. Cutolo, M. Giordano, and A. Cusano, "Refractive index sensor based on microstructured fiber Bragg grating," IEEE Photon. Technol. Lett. 17, 1250-1252 (2005).

[29] A. Iadicicco, A. Cusano, A. Cutolo, R. Bernini, and M. Giordano, "Thinned fiber Bragg gratings as high sensitivity refractive index sensor," IEEE Photon. Technol. Lett. 16, 1149-1151 (2004).

[30] R. Yang, J. Long, T. Yan-Nan, S. Li-Peng, L. Jie, and G. Bai-Ou, "High-efficiency ultraviolet inscription of Bragg gratings in microfibers," IEEE Photon. J. 4, 181-186 (2012).

[31] Y. Ran, Y.-N. Tan, L.-P. Sun, S. Gao, J. Li, L. Jin, and B.-O. Guan, "193nm excimer laser inscribed Bragg gratings in microfibers for refractive index sensing," Opt. Express 19, 18577-18583 (2011).

[32] F. Bilodeau, B. Malo, J. Albert, D. C. Johnson, K. O. Hill, Y. Hibino, M. Abe, and M. Kawachi, "Photosensitization of optical fiber and silica-on-silicon/silica waveguides," Opt. Lett. 18, 953-955 (1993).

[33] Y. Zhang, B. Lin, S. C. Tjin, H. Zhang, G. Wang, P. Shum, and X. Zhang, "Refractive index sensing based on higher-order mode reflection of a microfiber Bragg grating," Opt. Express 18, 26345-26350 (2010).

[34] F. Xu, G. Brambilla, and Y. Lu, "A microfluidic refractometric sensor based on gratings in optical fibre microwires," Opt. Express 17, 20866-20871 (2009).

[35] W. Ding, S. R. Andrews, T. A. Birks, and S. A. Maier, "Modal coupling in fiber tapers decorated with metallic surface gratings," Opt. Lett. 31, 2556-2558 (2006). 
[36] M. C. Phan Huy, G. Laffont, V. Dewynter, P. Ferdinand, P. Roy, J.-L. Auguste, D. Pagnoux, W. Blanc, and B. Dussardier, "Three-hole microstructured optical fiber for efficient fiber Bragg grating refractometer," Opt. Lett. 32, 2390-2392 (2007).

[37] F. Xu, G. Brambilla, J. Feng, and Y.-Q. Lu, "A microfiber Bragg grating based on a microstructured rod: a proposal," IEEE Photon. Technol. Lett. 22, 218-220 (2010).

[38] R. R. Gattass and E. Mazur, "Femtosecond laser micromachining in transparent materials," Nat. Photon. 2, 219-225 (2008).

[39] J. C. Knight, "Photonic crystal fibres," Natur 424, 847-851 (2003).

[40] M. Bayindir, F. Sorin, A. F. Abouraddy, J. Viens, S. D. Hart, J. D. Joannopoulos, and Y. Fink, "Metal-insulator-semiconductor optoelectronic fibres," Natur 431, 826-829 (2004).

[41] G. Brambilla, F. Xu, and X. Feng, "Fabrication of optical fibre nanowires and their optical and mechanical characterisation," Electron. Lett. 42, 517-519 (2006).

[42] W. Streifer and A. Hardy, "Analysis of two-dimensional waveguides with misaligned or curved gratings," Quantum Electronics, IEEE Journal of 14, 935-943 (1978).

[43] F. Xu and G. Brambilla, "Preservation of Micro-Optical Fibers by Embedding," Jpn. J. Apl. Phys. 47, 6675-6677 (2008).

[44] J.-1. Kou, Z.-d. Huang, G. Zhu, F. Xu, and Y.-q. Lu, "Wave guiding properties and sensitivity of D-shaped optical fiber microwire devices," Applied Physics B 102, 615-619 (2011).

[45] J.-L. Kou, S.-J. Qiu, F. Xu, Y.-Q. Lu, Y. Yuan, and G. Zhao, "Miniaturized MetalDielectric-Hybrid Fiber Tip Grating for Refractive Index Sensing," IEEE Photon. Technol. Lett. 23, 1712-1714 (2011).

[46] J. Canning, M. Stevenson, S. Bandyopadhyay, and K. Cook, "Extreme Silica Optical Fibre Gratings," Sensors 8, 6448-6452 (2008). 